\title{
CRISPR-CAS SYSTEM: A FEASIBLE SOLUTION FOR GETTING RID OF PERSISTENT VIRAL INFECTION?
}

\author{
Zhaohua Zhong \\ Department of Microbiology, Harbin Medical University, Harbin 150081, China
}

Many viruses, including DNA and RNA viruses, can persistently replicate in the infected cells and cause a prolonged damage. Herpes viruses can be latent in its host cells. Human Immunodeficiency Virus (HIV) reversely transcripts its RNA genome to DNA and integrates to the host's chromosome. Hepatitis B Virus (HBV) and Human Papilloma Virus (HPV) persistently stay in the host cells as an episomal form, their genomic DNAs can also be integrated to the host's chromosomes. Viral persistent infection is the major cause of the mortality among infectious diseases.

Conventional antiviral chemotherapy is effective only when the viruses are actively biosynthesized and duplicated, it does little if the viruses are latent or the viral genomes are integrated. RNA interference (RNAi)mediated gene silencing has been applied to intervene the viral biosynthesis. It targets to viral genomic RNA and mRNA, thus, cannot degrade the viral genomic DNA or proviral DNA. So far, no practical strategy is available to specifically eliminate the viral episomal and integrated DNAs from the infected cells. However, recently CRISPR-Cas system, a bacterially derived RNA-guided DNA editing system and a novel gene editing technology,makes it possible to get rid of the viral genomic DNA either in episomal or integrated forms.

In 1980s, scientists found that there are Clustered, Regularly Interspaced, Short Palindromic Repeats (CRISPR) in the genomes of bacteria and archaea. Recent studies showed CRISPR is a microbial adaptive antiviral immunity system. CRISPR expresses short RNAs that are complementary to a part of the genome sequence of bacterial virus. When bacteria are infected with viruses, the short CRISPR RNA bind to the viral genome through the complementary sequences and guide CRISPR-associated (Cas) endnucleases to cut the phage genome (Wiedenheft et al., 2012). The type II Cas endnuclease, Cas9, relies on two small RNAs, CRIPSR RNAs (crRNAs) and trans-activating crRNA (tracrRNA), to sequence-specifically silence viral genes (Jinek et al., 2012). Interestingly, crRNA and tracrRNA can be combined as a single guide RNA (sgRNA) with about 80 nucleotides in length (Jinek et al., 2012). A remarkable finding recently is that CRISPR-Cas system works well not only in the prokaryotic cells but also in the eukaryotic cells upon some modification of Cas9 protein derived from Streptococcus pyogenes (Mali et al., 2013). Thus, the bacterial originated CRISPR-Cas system became a hot gene editing tool from fruit fly to mouse last year (Hwang et al., 2013; Mali et al., 2013).

Despite of the applications such as gene knockout and transgenesis, CRISPR-Cas system has been attempted for intervening human virus infections. CRISPR-Cas system may be used to cleave viral DNAs in either episomal form or integrated form, as long as the sgRNA has been carefully designed to target a specific sequence in the viral genomic DNA. Indeed, studies showed that Cas9/sgRNAcan be adapted for disrupting the LTR elements of Human Immunodeficiency Virus (HIV) provirus and causing a significant loss of LTR-driven expression (Ebina et al., 2013). A similar study showed that using Cas9 and sgRNA targeting the LTR U3 region, a $9.7 \mathrm{~kb}$ fragment of the integrated HIV proviral DNA can be excised (Hu et al., 2014a). CRISPR-Cas system has also been used to interfere herpes virus infections (Wang and Quake, 2014; Bi et al., 2014). Exposure to a CRISPR-Cas9 vector targeted to Epstein-Barr virus genome can dramatically suppress the proliferation of the cells obtained from a patient with Burkitt's lymphoma and decrease the viral load (Wang and Quake, 2014). Study showed CRISPR-Cas system can restrict $\mathrm{HBV}$ infection under simulative in vivo infection scenario (Lin et al., 2014a). Studies also showed that CRISPR-Cas system works out in intervening Human Papilloma Virus (HPV) infection (Kennedy et al., 2014; Hu et al., 2014b; Zhen et al., 2014).

These studies proved in principle that CRISPR-Cas system is potentially feasible for treating persistent viral infection. Technically, using plasmid transfection or viral vector transduction, it is possible to cleave specific viral DNAs by expressing Cas9 and sgRNA in human body. Thus, Cas9/sgRNA can be a clinical therapeutic solution for getting rid of the persistency of many viruses.

However, there are challenges to cross the gap between laboratory benches and hospital beds before its clinical application. First, how to effectively deliver the 
Cas9/sgRNA-expressing vector to human body, especially, to ensure that the vectors reach every infected cell, is still technically difficult. Optimizing the delivery protocol will be a key factor for the translation of CRISPR-Cas system from benches to beds. Second, for the integrated viruses, the insertion of the viral DNA always distributes randomly in the host's genome, identification of an effective target sequence may be needed from patient to patient, that means the therapy would be very much individualized. Third, off-target is another concern for the application of CRISPR-Cas system in vivo. The specificity of Cas9 cleavage depends on a $20 \mathrm{nt}$ sequence in the sgRNA, theoretically, unexpected off-target effects is hard to avoid (Lin et al., 2014b). Well-designed and thoroughly tested sgRNA is needed before introducing it to human body. Finally, a variety of viruses can cause persistent infection, some of them have a DNA genome or a proviral DNA and the others do not (e.g., hepatitis C virus). CRISPR-Cas technique may not be applicable for these RNA viruses.

\section{References}

Bi, Y., L. Sun, D. Gao, C. Ding and Z. Li et al., 2014. High-efficiency targeted editing of large viral genomes by RNA-guided nucleases. PLoS Pathog., 10: e1004090-e1004090. DOI: 10.1371/journal.ppat.1004090

Ebina, H., N. Misawa, Y. Kanemura and Y. Koyanagi, 2013. Harnessing the CRISPR/Cas9 system to disrupt latent HIV-1 provirus. Sci. Rep., 3: 2510. DOI: $10.1038 /$ srep02510

$\mathrm{Hu}$, W., R. Kaminski, F. Yang, Y. Zhang and L. Cosentino et al., 2014a. RNA-directed gene editing specifically eradicates latent and prevents new HIV1 infection. Proc. Nat. Acad. Sci. USA., 111: 1146111466. DOI: 10.1073/pnas.1405186111

$\mathrm{Hu}, \mathrm{Z}$., L. Yu, D. Zhu, W. Ding and X. Wang et al., 2014b. Disruption of HPV16-E7 by CRISPR/Cas system induces apoptosis and growth inhibition in HPV16 positive human cervical cancer cells. BioMed. Res. Int., 2014: 612823-612831. DOI: 10.1155/2014/612823

Hwang, W.Y., Y. Fu, D. Reyon, M.L. Maeder and S.Q. Tsai et al., 2013. Efficient genome editing in zebrafish using a CRISPR-Cas system. Nat. Biotechnol., 31: 227-229. DOI: 10.1038/nbt.2501
Jinek, M., K. Chylinski, I. Fonfara, M. Hauer and J.A. Doudna et al., 2012. A programmable dual-RNAguided DNA endonuclease in adaptive bacterial immunity. Science, 337: 816-821. DOI: 10.1126/science. 1225829

Kennedy, E.M., A.V. Kornepati, M. Goldstein, H.P. Bogerd and B.C. Poling et al., 2014. Inactivation of the human papilloma virus E6 or E7 gene in cervical carcinoma cells using a bacterial CRISPR/Cas RNA-guided endonuclease. J. Virol. PMID: 25100830

Lin, S.R., H.C. Yang, Y.T. Kuo, C.J. Liu and T.Y. Yang et al., 2014a. The CRISPR/Cas9 system facilitates clearance of the intrahepatic HBV templates in vivo. Mol. Ther. Nucleic Acids, 3: e186-e186. DOI: $10.1038 / \mathrm{mtna} .2014 .38$

Lin, Y., T.J. Cradick, M.T. Brown, H. Deshmukh and P. Ranjan et al., 2014b. CRISPR/Cas9 systems have off-target activity with insertions or deletions between target DNA and guide RNA sequences. Nucleic Acids Res., 42: 7473-7485. DOI: 10.1093/nar/gku402

Mali, P., L. Yang, K.M. Esvelt, J. Aach and M. Guell et al., 2013. RNA-guided human genome engineering via Cas9. Science, 339: 823-826. DOI: 10.1126/science. 1232033

Wang, J. and S.R. Quake, 2014. RNA-guided endonuclease provides a therapeutic strategy to cure latent herpesviridae infection. Proc. Nat. Acad. Sci. USA. PMID: 25157128

Wiedenheft, B., S.H. Sternberg and J.A. Doudna, 2012. RNA-guided genetic silencing systems in bacteria and archaea. Nature, 482: 331-338. DOI: 10.1038/nature10886.

Zhen, S., L. Hua, Y. Takahashi, S. Narita and Y.H. Liu et al., 2014. In vitro and in vivo growth suppression of human papillomavirus 16-positive cervical cancer cells by CRISPR/Cas9. Biochem. Biophys. Res. Commun., 450: 1422-1426. DOI: 10.1016/j.bbrc.2014.07.014 